\title{
Growth optimization of mangrove Streptomyces for enhancement of actinorhodin production
}

\author{
Nur Hafizah Azizana, Nur Azam Amirdun@ Amirudin, Zarina Zainuddin ${ }^{\mathrm{b}}$, \\ Mohd Faez Sharifa, Ahmed Jalal Khan Chowdhury ${ }^{\mathrm{c}, *}$, Zaima Azira Zainal Abidin ${ }^{\mathrm{a}, *}$ \\ ${ }^{a}$ Department of Biotechnology, Kulliyyah of Science, International Islamic University Malaysia, Kuantan, 25200 Pahang, \\ Malaysia, email: zzaima@iium.edu.my (Z.A.Z. Abidin) \\ ${ }^{b}$ Department of Plant Science, Kulliyyah of Science, International Islamic University Malaysia, Kuantan, 25200 Pahang, Malaysia \\ 'Department of Marine Science, Kulliyyah of Science, International Islamic University Malaysia, Kuantan, 25200 Pahang, \\ Malaysia, email: jkchowdhury@iium.edu.my
}

Received 15 August 2020; Accepted 23 November 2020

\begin{abstract}
A B S T R A C T
The family Streptomycetaceae, notably species in genus Streptomyces, have long been the subject of investigation due to their well-known ability to produce secondary metabolites. Even though thousands of secondary metabolites have been discovered from Streptomyces, less attention has been given in understanding the bioprospecting of the secondary metabolites in Streptomyces living in mangrove ecosystem. Therefore, the current study was designed to investigate the production of actinorhodin from mangrove Streptomyces which was grown in different parameters. Phylogenetic analysis showed that the Streptomyces sp. K2-11 belongs to Streptomyces prasinus strain NRRL B-12521 with $99.62 \%$ similarity. High-performance liquid chromatography analysis has detected the presence of actinorhodin compound in Streptomyces sp. k2-11. The compound showed inhibitory activity to Bacillus subtilis and no activity against Streptococcus aureus based on disc diffusion assay. Streptomyces sp. growth in SMMS media was recorded to be the highest $\left(1.49 \times 10^{8} \mathrm{cfu} /\right.$ $\mathrm{mL}$ ) compared with SYE and R2 media. The production of actinorhodin was tested on three different parameters: incubation temperature, $\mathrm{pH}$ and glucose levels. The highest production of actinorhodin was recorded at $33^{\circ} \mathrm{C}\left(1.95 \times 10^{-6} \mathrm{~mol} / \mathrm{L}\right)$, pH $5\left(7.1 \times 10^{-6} \mathrm{~mol} / \mathrm{L}\right)$ and $50 \% \mathrm{w} / \mathrm{v}$ glucose $\left(9.56 \times 10^{-6} \mathrm{~mol} / \mathrm{L}\right)$ for $60 \mathrm{~h}$ of incubation. The present finding suggested that mangrove Streptomyces sp. K2-11 produced high yield of actinorhodin under extreme or stress condition.
\end{abstract}

Keywords: Actinorhodin; Antibiotic; Mangrove; Secondary metabolites; Streptomyces

\section{Introduction}

Streptomycetes are filamentous, Gram-positive bacteria that exist in a variety of soils, compost, plants and water. These microorganisms also are known as a producer of many secondary metabolites, such as antibiotics. Among the actinomycetes, about 7,600 compounds are produced by Streptomyces species. These metabolites are known to have antibacterial, antifungal, antioxidant, neuritogenic, anti-cancer, anti- algal, anti-helmintic, anti-malarial and anti-inflammatory activities [1]. They exhibit a range of life cycles, which are unique among the prokaryotes, and appear to play a major role in the recycling of organic matter in the soil ecosystem [2].

It is commonly known that the production of secondary metabolites from microorganisms, such as antibiotics,

* Corresponding authors. 
is the useful mechanism to protect themselves from foreign organisms. The same mechanism was used by humans to produce antibiotics that can kill infection of microorganisms on human body. The uses of antibiotics are increasing worldwide due to population growth, and overuse lead to increasing antibiotic resistance, which raises the price of healthcare products and equipment [3]. The decrease of antibiotic effectiveness contributes to major threat for medical sector. In the United States alone, around 23,000 people die each year as a result of antibiotic resistance infections [4]. Therefore, research on the production of antibiotic is vital to improve the effectiveness of medicine especially those that are related to microbial infection.

The production of secondary metabolites usually coincides with their pathway-specific regulatory genes, which are frequently expressed at low level. The low level effective concentration and remarkable pleiotropic effects usually complicates the regulatory cascades that govern this process. Consequently, the possibility of over-expressing the functional protein (secondary metabolic gene cluster) that is bound to produce the useful compound is predicted to favor the production of the secondary metabolite. Thus, this research was conducted to enhance the production of actinorhodin from Streptomyces through growth optimization, which involved various growth parameters and conditions.

\section{Methodology}

\subsection{Bacterial strain}

Streptomyces sp. K2-11 was previously isolated from mangrove forest of Tanjung Lumpur, Kuantan, Pahang. Preliminary screening showed antibacterial potential of Streptomyces sp. K2-11 against B. subtilis. Streptomyces sp. K2-11 was maintained on an SYE agar (starch-yeast extract).

\subsection{Molecular identification of streptomycetes}

Universal primers of $16 \mathrm{sF}$ (5'-AGAGTTTGATCCTGGCT CAG-3') and 16sR (5'-AAG GAG GTGATCCAGCCGCA-3') were used to target the conserved region of $16 \mathrm{~S}$ rRNA to amplify an approximately $1.5 \mathrm{~kb}$ long DNA fragment. Amplification was performed in a final volume of $50 \mu \mathrm{L}$, which consist of $200 \mathrm{ng}$ of template genomic DNA, $0.4 \mu \mathrm{M}$ of each primer, $25 \mu \mathrm{L}$ of $2 \times$ MyTaq $^{\mathrm{TM}}$ Mix (Bioline, UK) and sterile ultrapure water. The $16 \mathrm{~S}$ rRNA gene fragment amplification reaction was carried out in an automated thermal cycler (Eppendorf Mastercycler Gradient, Germany) with initial denaturation and denaturation performed at $94^{\circ} \mathrm{C}$ for $5 \mathrm{~min}$ and $30 \mathrm{~s}$, respectively, annealing temperature at $55^{\circ} \mathrm{C}$ for $60 \mathrm{~s}$, extension at $72^{\circ} \mathrm{C}$ for $2 \mathrm{~min}$ (30 cycles) and final extension at $72^{\circ} \mathrm{C}$ for $10 \mathrm{~min}$. The PCR products were examined and visualized with $1.0 \%(\mathrm{w} / \mathrm{v})$ agarose gel electrophoresis, separated at $70 \mathrm{~V}$ for $45 \mathrm{~min}$. The product was purified and submitted for sequencing.

\subsection{Phylogenetic analysis}

The 16S rRNA sequences were manually verified using Sequence Scanner software version 2.0 (Applied Biosystems, ThermoFisher Scientific). The sequences were assembled using the Bioedit Sequence Alignment Editor program. The sequences were matched with the GenBank database using BLASTn (National Center for Biotechnology Information) search tool [5]. The 16S rRNA gene sequences of the isolates and their closest phylogenetic neighbors were aligned and evaluated for their homology using CLUSTALW module. Phylogenetic tree was generated using the Molecular Evolutionary Genetics Analysis (MEGA) version 6.0 [6]. The tree topology was evaluated in a bootstrap analysis based on 1,000 replicates using the neighbor joining algorithm [7].

The nucleotide sequence of the isolate was deposited in the GenBank database via the web-based submission data wizard (http://www.ncbi.nlm.nih.gov/home/submit-wizard. shtml).

\subsection{Actinorhodin extraction}

Streptomyces K2-11 was grown in SMMS media. After $7 \mathrm{~d}$ of incubation, actinorhodin was extracted with $2 \mathrm{~L}$ of $3 \mathrm{~N}$ $\mathrm{KOH}$ from a $2-\mathrm{L}$ sample. The suspension was centrifuged at $4,000 \times \mathrm{g}$ for $10 \mathrm{~min}$ and the pellet was removed.

\subsection{HPLC analysis}

Extracted supernatant was subjected to reversed-phase HPLC analysis (PerkinElmer) under the following conditions: column, Phenomenex LunaC18 (2) (4.6 i.d. $150 \mathrm{~mm}$, Waters); column temperature, $40^{\circ} \mathrm{C}$; gradient elution with mobile phase comprised of solvent A (acetonitrile, containing $0.5 \%$ acetic acid), and solvent $\mathrm{B}$ (0.5\% acetic acid) (Analytical Laboratory, Kulliyyah of Science, International Islamic University Malaysia). Gradient profile, 0-20 min: 43.5\%A, 20-25 min: 43.5\%-60\%A, 25-28 min: 60\%-95\%A, 28-32 $\mathrm{min}: 95 \% \mathrm{~A}, 32-35 \mathrm{~min}: 95 \%-43.5 \% \mathrm{~A}$; flow rate, $1.0 \mathrm{~mL} / \mathrm{min}$; and detection of 250 and $600 \mathrm{~nm}$ using a photo-diode array detector (PD-8020, TOSOH).

\subsection{Antimicrobial activity screening}

Determination of antimicrobial activity of actinorhodin was performed using a standard antimicrobial susceptibility test. Two test organisms consisted of two Gram-positive bacteria (Bacillus subtilis IMR B 144/11 C and Staphylococcus aureus ATCC 259233), which were obtained from the laboratory collection.

The antimicrobial activity of the ethyl acetate extracts was assessed using modified Kirby-Bauer disc diffusion method as described by Wanger [8]. The antibiotic extract of the isolates impregnated onto sterile $6 \mathrm{~mm}$ in diameter blank antibiotic disc (Oxoid, UK). The concentration of antibiotics was adjusted to $1 \mathrm{mg} / \mathrm{mL}$ and $20 \mu \mathrm{L}$ was injected onto the discs. The disc was then left to dry and placed on the MHA surface seeded with $100 \mu \mathrm{L}$ test organisms. Standard antibiotic chloramphenicol (10 $\mu$; Oxoid, UK) discs were used as positive control for bacteria, while disc impregnated with methanol was taken as negative control. The plates were incubated at $30^{\circ} \mathrm{C}(48 \mathrm{~h})$. The antimicrobial activity was determined by measuring the diameter of the inhibition zone (expressed in $\mathrm{mm}$ ), where the colonies are failed to grow. The test was conducted in triplicate and results were expressed as mean \pm SD (standard deviation). 


\subsection{Streptomycetes growth determination} on different growth media

Streptomyces sp. K2-11 were grown in all types of broth (SMMS, SYE and R2; $500 \mathrm{~mL}$ ). As much as $1 \mathrm{~mL}$ of culture broth were taken from each conical flask after 5, 10, 20, 30, 40,50 and $60 \mathrm{~h}$ and were transferred into $1.5 \mathrm{~mL}$ cuvette. The optical density of Streptomyces was determined by using spectrophotometer at wavelength $600 \mathrm{~nm}$. This step was repeated thrice and the results were recorded.

A series of dilution were prepared from $10^{-1}$ to $10^{-9}$-fold and a streak plate technique was performed for dilution $\left(10^{-6}, 10^{-8}\right.$ and $10^{-9}$-fold $)$. All plates were incubated at $30^{\circ} \mathrm{C}$ and the growth of the colony was observed and recorded.

\subsection{Actinorhodin production optimization}

Streptomyces sp. K2-11 was cultured in SMMS broth with different conditions and parameters applied in order to optimize the production of actinorhodin. The parameters were temperature $\left(25^{\circ} \mathrm{C}, 27^{\circ} \mathrm{C}, 30^{\circ} \mathrm{C}, 33^{\circ} \mathrm{C}\right.$ and $\left.37^{\circ} \mathrm{C}\right), \mathrm{pH}$ $(\mathrm{pH} 3, \mathrm{pH} 5, \mathrm{pH} 7, \mathrm{pH} 9$ and $\mathrm{pH} 12)$, and glucose concentration $(5 \%(\mathrm{w} / \mathrm{v}), 25 \%(\mathrm{w} / \mathrm{v}), 50 \%(\mathrm{w} / \mathrm{v}), 75 \%(\mathrm{w} / \mathrm{v})$ and $95 \%$ $(\mathrm{w} / \mathrm{v}))$.

Fresh Streptomyces sp. K2-11 was inoculated into five labelled 500-mL conical flasks containing $200 \mathrm{~mL}$ SMMS broth with $\mathrm{pH}$ was adjusted to 7 with hydrochloric acid and sodium hydroxide. The broth containing Streptomyces was shaken gently. Then, the broth was incubated into five different temperature that at $25^{\circ} \mathrm{C}, 27^{\circ} \mathrm{C}, 30^{\circ} \mathrm{C}, 33^{\circ} \mathrm{C}$ and $37^{\circ} \mathrm{C}$. Actinorhodin was extracted at 5, 10, 20, 30, 40, 50 and $60 \mathrm{~h}$.

Another fresh Streptomyces sp. K2-11 was inoculated independently with $200 \mathrm{~mL}$ SMMS media $\mathrm{pH}$ adjusted to $\mathrm{pH} 3, \mathrm{pH} \mathrm{5,} \mathrm{pH} \mathrm{7,} \mathrm{pH} 9$ and $\mathrm{pH}$ 12. The broths were incubated at $30^{\circ} \mathrm{C}$ with agitation at $150 \mathrm{rpm}$. The inoculating step was repeated in SMMS broth with customised glucose concentration 5\% (w/v), 25\% (w/v), 50\% (w/v), 75\% (w/v) and $95 \%(\mathrm{w} / \mathrm{v})$. The $\mathrm{pH}$ was adjusted to 7 and incubated at $30^{\circ} \mathrm{C}$. The antibiotics were then extracted after incubation. The concentration of antibiotics from each parameter was calculated and analysed.

\subsection{Actinorhodin quantification}

The absorbance of actinorhodin was measured by using spectrophotometer at $640 \mathrm{~nm}$. The actinorhodin concentration was calculated based on an extinction coefficient of $25,320 \mathrm{M}^{-1} \mathrm{~cm}^{-1}$ [9].

\subsection{Statistical analysis}

All data were recorded and analysed statistically using Microsoft Excel $^{\odot} 2010$ edition and IBM $^{\odot}$ SPSS Statistics 21.0. In order to understand the relationship among the variables (incubation temperature, $\mathrm{pH}$ level and glucose level) or the degree of association between the two, correlation model (Pearson correlation) was adopted. Once the correlation was successfully computed, the probability that observed correlation occurred by chance was determined by conducting significance test. The data were grouped according different parameters (incubation temperature, $\mathrm{pH}$ level and glucose level). Each parameter was divided into five different degrees and levels. The concentration of antibiotics from each level was taken at 5, 10, 20, 30, 40, 50 and $60 \mathrm{~h}$ of incubation. Thus, the sample size each parameter was $n=40$ (including $0 \mathrm{~h}$ as zero antibiotic concentration was indicated as a value).

\section{Result and discussion}

\subsection{Phylogenetic analysis}

BLAST analysis on 16s ribosomal RNA gene sequence revealed that Streptomyces sp. K2-11 is highly similar to Streptomyces prasinus strain NRRL B-12521 (Fig. 1) with $99.62 \%$ similarity.

Streptomyces prasinus previously known as Streptomyces bambergiensis, commonly found as soil inhabitant and was first found by Wallhausser et al. [10] from soil of Bamberg, Germany. Streptomyces prasinus was reported to produce secondary metabolites, which were prasinomycin, prasinons A and B [11,12]. Thus far, no study has been reported on the production of actinorhodin by Streptomyces prasinus.

\subsection{HPLC analysis}

The HPLC analysis has successfully detected actinorhodin at retention time $25 \mathrm{~min}$ at $420 \mathrm{~nm}$ absorbance using combination of gradient acetonitrile and $0.1 \%$ acetic acid (Fig. 2). Taguchi et al. [13] reported that in vitro reconstitution systems of the actinorhodin biosynthetic pathway leading to (S)-DNPA had successfully detected and purified actinorhodin at a retention time of $25 \mathrm{~min}$ with absorbance at $420 \mathrm{~nm}$.

\subsection{Antimicrobial activity screening}

The biopotential of Streptomyces sp. K2-11 with respect to their antimicrobial properties for actinorhodin was

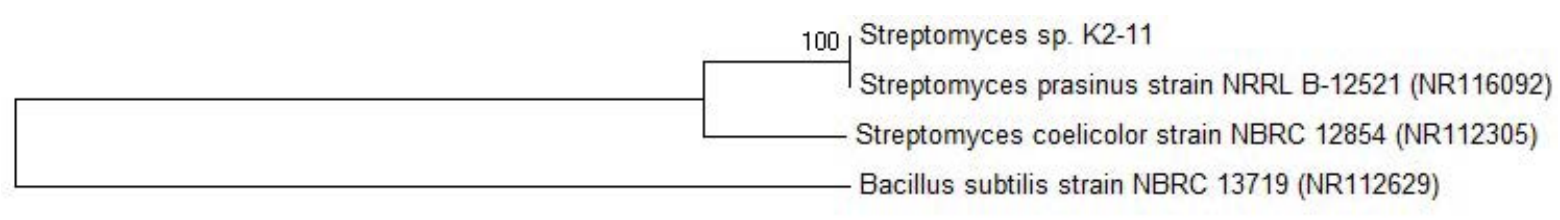

0.02

Fig. 1. Phylogenetic tree based on partial 16s rRNA sequence using neighbor-joining algorithm (Tamura-Nei 93 parameter model). Bacillus subtilis NBRC 13719 was used as an outgroup. 


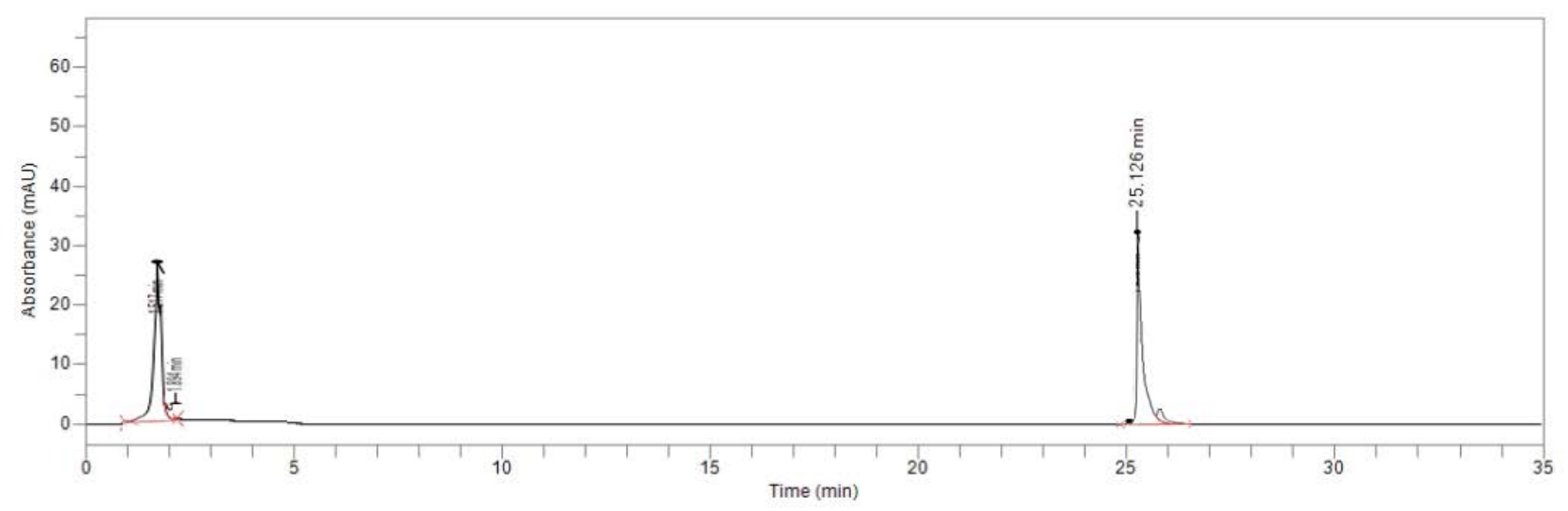

Fig. 2. HPLC profile for actinorhodin from Streptomyces sp. K2-11.

further evaluated for its antagonistic potential against two test organisms (two Gram-positive bacteria) using disc diffusion method.

Actinorhodin showed antibacterial activity against Bacillus subtilis IMR B 144/11 C $(8.0 \pm 0.2 \mathrm{~mm})$, but no antibacterial activity was observed against of $S$. aureus ATCC 259233 (Table 1). According to Mak [14], the activity against S. aureus ATCC 259233 is intriguing because this strain is reported to be resistant to ampicillin, amoxicillin/clavulanic acid, ciprofloxacin, cephalothin, doxycycline, gentamicin, erythromycin, imipenem, methicillin, penicillin, tetracycline, oxacillin, azithromycin, clindamycin, ceftriaxone, rifampin, amikacin and tobramycin, suggesting that actinorhodin can bypass this resistance. The absence of inhibition zone against of $S$. aureus in this study was probably due to the presence of different structure of actinorhodin [15]. Therefore, further investigation concerning actinorhodin produced by Streptomyces sp. K2-11 needs to be conducted in future.

\subsection{Streptomyces $s p$. K2-11 growth determination on different growth media}

According to Fig. 3, Streptomyces sp. K2-11 exhibited different exponential phase in different growth media. In SMMS, the growth reached exponential phase after $5 \mathrm{~h}$ of incubation, while in R2 and SYE the exponential phase was between 10 and $20 \mathrm{~h}$, respectively. Streptomyces sp. K2-11 recorded the highest growth $\left(9.50 \times 10^{6} \mathrm{CFU} / \mathrm{mL}\right)$ in SMMS, followed $3.43 \times 10^{6} \mathrm{CFU} / \mathrm{mL}$ in $\mathrm{R} 2$ and $2.43 \times 10^{6} \mathrm{CFU} / \mathrm{mL}$ in SYE.

\subsection{Antibiotic production optimization}

Based on Fig. 4, actinorhodin was produced considerably high at $33^{\circ} \mathrm{C}$ in $60 \mathrm{~h}$ of incubation with count of $1.95 \times 10^{-6} \mathrm{~mol} / \mathrm{L}$. The lowest concentration of actinorhodin was recorded at $27^{\circ} \mathrm{C}\left(1.18 \times 10^{-7} \mathrm{~mol} / \mathrm{L}\right)$ in $10 \mathrm{~h}$ of incubation. Statistical data showed that if one variable change (media and incubation time), it will give significant change to the CFU/mL of the Streptomyces sp. K2-11. Analysed data revealed that there was no significant relationship between temperature and actinorhodin production but
Table 1

Antimicrobial activities of actinorhodin from Streptomyces sp. K2-11 using disc diffusion method

\begin{tabular}{lll}
\hline & \multicolumn{2}{c}{ Activity against $^{a}(\mathrm{~mm})$} \\
\hline Antibiotic & Bacillus subtilis & Staphylococcus aureus \\
\hline Actinorhodin & $8.0 \pm 0.2$ & - \\
Chloramphenicol & $25.5 \pm 0.6$ & $24.2 \pm 0.6$ \\
\hline
\end{tabular}

${ }^{a}$ Estimated by measuring the diameter of the clear zone of inhibition produced. Values are recorded as mean of three replications \pm standard deviation.

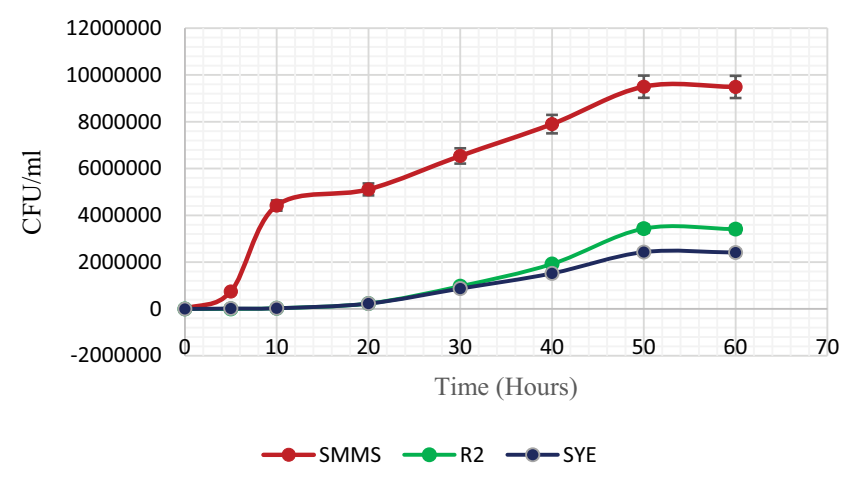

Fig. 3. Colony forming unit over time of Streptomyces in different media.

there was significant change of actinorhodin production over incubation time of Streptomyces sp. K2-11.

According to Wang et al. [16], the production of actinorhodin was highly depended on $\mathrm{pH}$ level. TThe shift of $\mathrm{pH}$ level of Streptomyces growth environment consequently effect the production of antibiotics [16-18]. Hence, this work has studied the correlation between $\mathrm{pH}$ and actinorhodin concentration. The calculation nevertheless has revealed a weak relationship between different $\mathrm{pH}$ level and actinorhodin production. Based on Fig. 5, actinorhodin was produced the highest $\left(7.1 \times 10^{-6} \mathrm{~mol} / \mathrm{L}\right)$ at $\mathrm{pH} 5$ after $60 \mathrm{~h}$ of incubation. The lowest concentration 


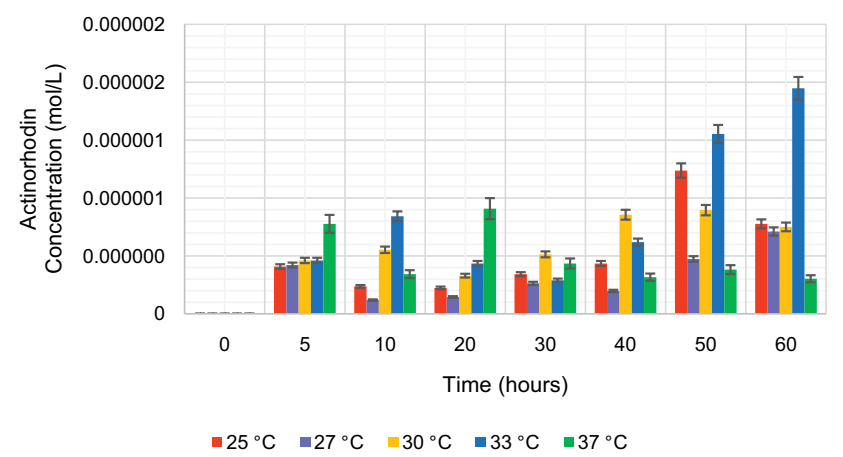

Fig. 4. Actinorhodin concentration production over time in different incubation temperature.

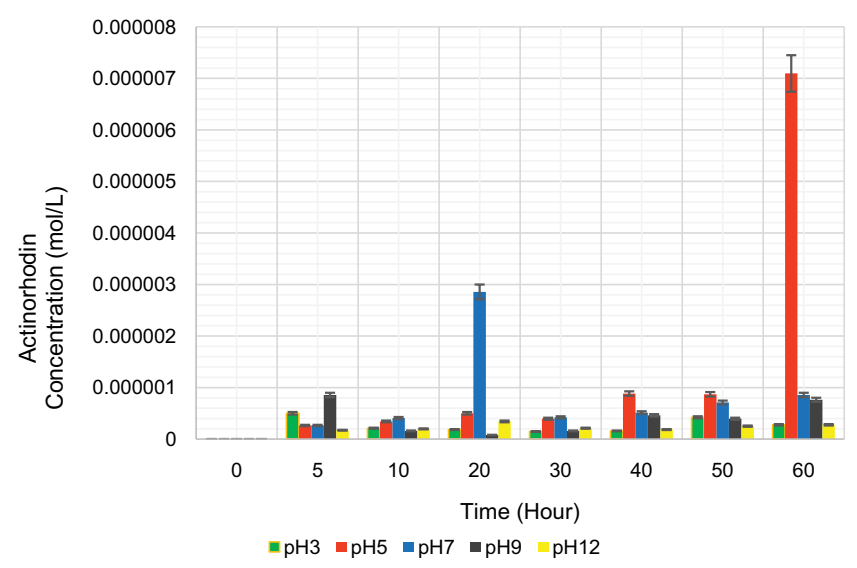

Fig. 5. Actinorhodin concentration production over time in different $\mathrm{pH}$ level.

of actinorhodin $\left(7.80 \times 10^{-8} \mathrm{~mol} / \mathrm{L}\right)$ was recorded at $\mathrm{pH}$ 9 after $20 \mathrm{~h}$ of incubation. According to Cihák et al. [19], actinorhodin compounds (actinorhodinic acid and $\gamma$-actinorhodin) were detected around $5.5 \mathrm{pH}$.

Velayudham ans Murugan [20] mentioned that different glucose concentration will affect the production of antibiotics. El-sayed et al. [21] also added that altering glucose concentration in fed batch culture can yield maximum production of natamycin, produced from Streptomyces natalensis. However, some antibiotics were produced in scarce in a certain condition of Streptomyces growth. In a carbon depleted state, Streptomyces secrete secondary metabolite, which is mostly antibiotic, as a response mechanisms [22]. Accordingly, this work examined the effect on actinorhodin concentration as a function of incubation time at various levels of glucose concentrations. The study disclosed that actinorhodin production was the highest when nurtured with $50 \% \mathrm{w} / \mathrm{v}$ glucose concentration at $60 \mathrm{~h}$ $\left(9.55 \times 10^{-7} \mathrm{~mol} / \mathrm{L}\right)$. Meanwhile in the presence of $75 \% \mathrm{w} / \mathrm{v}$ of glucose, the concentration of actinorhodin was at its lowest count $\left(2.50 \times 10^{-7} \mathrm{~mol} / \mathrm{L} ; 10 \mathrm{~h}\right.$ of incubation; Fig. 6$)$.

Statistical study shows that the production of actinorhodin had a significant relationship with incubation times in different glucose concentration. Forero et al. [23] reported that actinorhodin production was found to be strongly suppressed by the presence of high glucose concentration



Fig. 6. Actinorhodin concentration production over time in different glucose level.

in Streptomyces coelicolor. When the used carbon source glucose was depleted, the $S$. coelicolor produced significant actinorhodin concentration.

\section{Conclusion}

Streptomyces sp. K2-11 was identified to have $99.62 \%$ similarity with Streptomyces prasinus strain NRRL-B 12521. Actinorhodin produced by Streptomyces sp. K2-11 has successfully detected by HPLC was proven very efficient on the Bacillus subtilis growth. In addition, Streptomyces sp. K2-11 growth was nourished in SMMS medium rather on SYE and R2 media. Finally and most importantly, manipulation of parameters $(\mathrm{pH}$, temperature, glucose) has remarkably influenced actinorhodin yield in this study.

\section{Acknowledgment}

The authors would like to thank the Ministry of Higher Education Malaysia and Research Management Centre (RMC) of International Islamic University Malaysia for funding this project. Project ID: RACER19-046-0046 (2019).

\section{References}

[1] T.R.P. Kekuda, K.S. Shobha, R. Onkarappa, Studies on antioxidant and anthelmintic activity of two Streptomyces species isolated from Western Ghat soils of Agumbe, Karnataka, J. Pharm. Res., 3 (2010) 26-29.

[2] D. Coleman, M. Callaham, D. Crossley, Jr., Fundamentals of Soil Ecology, Academic Press, 2017.

[3] C.L. Ventola, The antibiotic resistance crisis: part 1: causes and threats, Pharm. Ther., 40 (2015) 277.

[4] Rate Review Annual Report, United States Department of Health and Human Services, USA, 2013.

[5] National Centre for Biotechnology Information (NCBI), PubChem Database. Available at: htttp:// www.ncbi.mlm.nih. gov. (accessed on June 12, 2019).

[6] K. Tamura, G. Stecher, D. Peterson, A. Filipski, S. Kumar, MEGA6: molecular evolutionary genetics analysis version 6.0, Mol. Biol. Evol., 30 (2013) 2725-2729.

[7] N. Saitou, M. Nei, The neighbour-joining method: a new method for reconstructing phylogenetic trees, Mol. Biol. Evol., 4 (1987) 406-425.

[8] A. Wanger, In: R. Schwalbe, L.S. Moore, A.C. Goodwin, Eds., Antimicrobial Susceptibility Testing Protocols, CRC Press, 2007, pp. 53-73. 
[9] I. Borodina, J. Siebring, J. Zhang, C.P. Smith, G.V. Keulen, L. Dijkhuizen, J. Nielsen, Antibiotic overproduction in Streptomyces coelicolor A3 (2) mediated by phosphofructokinase deletion, J. Biol. Chem., 283 (2008) 25186-25199.

[10] K.H. Wallhausser, G. Nesemann, P. Prave A. Steigler, Moenomycin, a new antibiotic. I. Fermentation and isolation, Antimicrob. Agents Chemother., 5 (1965) 734-736.

[11] F.L. Weisenborn, J.L. Bouchard, D. Smith, F. Pansy, G. Maestrone, G. Miraglia, E. Meyers, The prasinomycins: antibiotics containing phosphorus, Nature, 213 (1967) 1092

[12] S.J. Box, M. Cole, G.H. Yeoman, Prasinons A and B: Potent insecticides from Streptomyces prasinus, Appl. Environ. Microbiol., 26 (1973) 699-704.

[13] T. Taguchi, T. Awakawa, Y. Nishihara, M. Kawamura, Y. Ohnishi, K. Ichinose, Bifunctionality of ActIV as a CyclaseThioesterase revealed by in vitro reconstitution of actinorhodin biosynthesis in Streptomyces coelicolor A3 (2), ChemBioChem, 18 (2017) 316-323.

[14] S.H.C. Mak, The Molecular Action of Actinorhodin, An Antibiotic Produced by Streptomyces coelicolor, Doctoral dissertation, 2017

[15] B.A. Rudd, D.A. Hopwood, Genetics of actinorhodin biosynthesis by Streptomyces coelicolor A3 (2), Microbiology, 114 (1979) 35-43.

[16] Y. Wang, X. Fang, Y. Cheng, X. Zhang, Manipulation of pH shift to enhance the growth and antibiotic activity of Xenorhabdus nematophila, J. Biomed. Biotechnol., (2011) 1-9.

[17] I.L. Bartek, M.J. Reichlen, R.W. Honaker, R.L. Leistikow, E.T. Clambey, M.S. Scobey, A.B. Hinds, S.E. Born, C.R. Covey, M.J. Schurr, A.J. Lenaerts, M.I. Voskuil, Antibiotic bactericidal activity is countered by maintaining $\mathrm{pH}$ homeostasis in Mycobacterium smegmatis, Am. Soc. Microbiol., 1 (2016) e00176-16.
[18] P. Grenni, V. Ancona, A.B. Caracciolo, Ecological effects of antibiotics on natural ecosystems: a review, Microchem. J., 136 (2018) 25-39.

[19] M. Cihák, Z. Kameník, K. Šmídová, N. Bergman, O. Benada O. Kofronová, K. Petrícková, J. Bobek, Secondary metabolites produced during the germination of Streptomyces coelicolor, Front. Microbiol., 8 (2017) 1-13.

[20] S. Velayudham, K. Murugan, Sequential optimization approach for enhanced production of antimicrobial compound from Streptomyces rochei BKM-4, South Indian J. Biol. Sci., 1 (2015) 72-79.

[21] S.A.E. El-sayed, M.A. Rizk, N. Yokoyama, I. Igarashi, Evaluation of the in vitro and in vivo inhibitory effect of thymoquinone on piroplasm parasites, Parasites Vectors, 12 (2019) 1-10.

[22] A.V.D. Meij, S.F. Worsley, M.I. Hutchings, G.P.V. Wezel, Chemical ecology of antibiotic production by actinomycetes, FEMS Microbiol. Rev., 41 (2017) 392-416.

[23] A. Forero, M. Sánchez, A. Chávez, B. Ruiz, R. Rodríguez-Sanoja, L. Servín-González, S. Sánchez, Possible involvement of the sco2127 gene product in glucose repression of actinorhodin production in Streptomyces coelicolor, Can. J. Microbiol., 58 (2012) 1195-1201. 